\title{
CONSTRUCTIVISMO RADICAL, MARCO TEÓRICO DE INVESTIGACIÓN Y ENSENANZA DE LAS CIENCIAS
}

\author{
MARTÍNEZ DELGADO, ALBERTO \\ IES Tartesos de Carnas. Sevilla
}

\begin{abstract}
SUMMARY
The article on radical constructivism criticizes its claim of being an original paradigm as well as criticizing some contradictions and scarcely justified theoretical leaps, and some specific problems over previous ideas in teaching and theoretical frames in scientific research.

The critique underlines the controversial subject of empirical and experimental methods in learning and research and proposes a comparison between empiricism and radical constructivism axioms (with a suggested isomorphism between supposed opposing theories) as well as a tendency for both paradigms to slip towards epistemological realism. Also discussed are some aspects regarding research management and education (LOGSE).
\end{abstract}

\section{LA EPISTEMOLOGÍA DEL CONSTRUCTI- VISMO RADICAL}

Dentro de la amplia diversidad de concepciones consideradas como constructivistas (Gómez-Granell, Coll Salvador, 1994, p. 8), centraremos nuestra atención en el constructivismo radical y en sus derivaciones para la enseñanza de las ciencias y para la investigación científica, por tratarse de una de las tendencias constructivistas de mayores aspiraciones teóricas y de mayor influencia en los medios académico-educativos. Dedicaremos especial atención a los planteamientos de Glasersfeld, (1987, 1990, 1991, 1993, 1995,1996), como representante ampliamente reconocido, tanto por la envergadura de sus planteamientos teóricos como por su impacto en la enseñanza de las ciencias y de las matemáticas (Ernest, 1989, p. 151; Kilpatrick, 1990, p. 39; Ernest, 1996, p. 79).

Los principios fundamentales del constructivismo radical han sido formulados, precisando la diferencia con el constructivismo trivial, por Glasersfeld (1991, p. 31):

a) El conocimiento no es recibido pasivamente sino construido activamente por el sujeto cognoscente;

b) La función de la cognición es adaptativa y sirve a la organización del mundo experiencial, no al descubrimiento de una realidad ontológica.

Aceptar sólo el primer principio es considerado como constructivismo trivial por los que aceptan ambos principios, porque este principio ha sido conocido desde Sócrates y, sin la ayuda del segundo, se mete de cabeza en todos los perennes problemas de la epistemología occidental.

Una caracterización similar del constructivismo radical puede encontrarse en Etchenberger y Shaw (1992, p. 411), en Saunders, (1993, p. 136) y en Noddings, (1990), quien sintetiza: «el conocimiento y la realidad misma son construcciones de la mente» (p. 14). El mismo Glasersfeld deja claro que el núcleo del constructivismo radical va más allá de una mera negación del conocimiento metafísico del mundo exterior, estable- 
ciendo una insalvable barrera entre el sujeto y los objetos exteriores y desplazando así al mundo exterior la caja negra, en que el conductismo había colocado los procesos internos del individuo. Así, Glasersfeld (1995, p. 114) concreta más el segundo principio (distintivo) del constructivismo radical:

«El segundo principio es que [...] el conocimiento no constituye una «pintura» del mundo. No representa en absoluto el mundo -comprende esquemas de acción, conceptos y pensamientos y distingue los que son considerados ventajosos de los que no lo son [...]-. De esto se sigue que lo que ordinariamente llamamos «hechos» no son elementos de un mundo independiente del observador, sino elementos de la experiencia de un observador.»

El constructivismo radical se presenta, por lo tanto, como un paradigma epistemológico contrapuesto al realismo, para el que la existencia de un mundo exterior al sujeto y con influencia en éste resulta innegable, y no sólo como eliminador de tentativas del individuo. La alternativa constructivista, que entronca con el idealismo filosófico tradicional, se postula presentando a su oponente realista de manera un tanto caricaturesca, identificándolo con formas metafísicas del materialismo pasivo o contemplativo que ya denunciara Marx en las «tesis sobre Feuerbach» (Marx, K., 1845/1975, p. 426), al mismo tiempo que proclama la grandilocuente pretensión de constituir una revolución mental respecto a los últimos dos mil quinientos años de historia de la humanidad (Glasersfeld, 1995, p. 19, p. 113).

\section{CONSTRUCTIVISMO RADICAL Y REALIS- MO.ISOMORFISMOFORMAL Y RECAIDAS EN EL REALISMO}

El anhelo de originalidad revolucionaria del constructivismo, sin embargo, encuentra serios problemas. Por un lado, es abusivo reducir el realismo, y el materialismo, a sus versiones mecanicistas, metafísicas y contemplativas. Por otra parte, el constructivismo no es la primera teoría que combate la metafísica. Ya el empirismo y el positivismo se opusieron a la concepción metafísica, con argumentos similares a los que en la actualidad emplea el constructivismo en contra del empirismo y del positivismo. La originalidad del constructivismo radical puede verse cuestionada por la coincidencia entre los postulados del constructivismo radical y los del empirismo de Hume (1734/1984) y por su posible subsunción dentro de los principios del positivismo (Martínez Delgado, 1995, p. 5).

El desplazamiento, por parte del constructivismo radical, de la fuente de las regularidades que dan lugar al conocimiento, desde el mundo exterior de los realistas (y especialmente de los realistas contemplativos) al sujeto constructor, puede esquematizarse como la «reconstrucción» de algunos conceptos tradicionales como «conocimiento, verdad, comunicación y comprensión» (Glasersfeld, 1995, p. 19). Esta reconstrucción, con cierta frecuencia, se reduce a la mera sustitución formal de términos como observar, objetos exteriores, objetivo aprendizaje por descubrimiento..., característicos de la teoría realista, por los correspondientes a construir (o inventar), mundo experiencial, viable, aprendizaje por invención..., más acordes con la orientación constructivista.

Esta relación formal entre el constructivismo y el realismo, sobre todo en la versión pasiva de éste, permite plantear la hipótesis de la existencia de un isomorfismo entre el modelo constructivista (con sus objetos y operaciones como constructos) y el modelo realista pasivo (con sus objetos y operaciones surgidos del mundo exterior). Esta hipotética relación de isomorfismo hace superfluo, en alguna medida y desde un punto de vista formal el problema del antagonismo entre los dos paradigmas, aunque no elimina la disyuntiva entre ellos, sobre todo si salimos del estrecho marco de las estructuras formales.

En los mismos planteamientos constructivistas radicales, es frecuente, por otra parte, una recaída en formulaciones realistas -de entrada, merece destacarse que el constructivismo se justifica como una verdad, o si se prefiere viabilidad, superior a la que pueda representar el realismo epistemológico-. Esta deriva hacia el realismo, en parte atribuible a la conformación realista de nuestro lenguaje (Glasersfeld, 1995, p. 19), quizás tenga raíces más profundas, como sugiere la historia del empirismo y el positivismo, formulados inicialmente bajo principios equivalentes a los del constructivismo y a los que el mismo constructivismo acusa actualmente de defender posiciones metafísicas y principios epistemológicos realistas. El vuelco evolutivo del empirismo y del positivismo hacia el realismo es revelador de la resistencia del paradigma realista a ser eliminado como perspectiva epistemológica, renuncia que quizás pueda ser explicada, en un enfoque marxista-althuseriano (Althusser, 1975, p. 101-102), como resultado de la contradicción, dentro de la «filosofía espontánea de los científicos», entre el elemento "intracientífico" [...] de carácter materialista y objetivista»y el elemento «"extracientífico" [...] reflejo sobre la práctica científica de tesis filosóficas elaboradas fuera de esta práctica [...] que, explotando las ciencias, sirven acríticamente a determinados objetivos que pertenecen a ideologías prácticas».

\section{LAS IDEAS PREVIAS EN LA ENSEÑANZA- APRENDIZAJE Y COMO MARCO TEORICO EN LA INVESTIGACION CIENTÍFICA}

A pesar de que algunos autores consideran que «el constructivismo no es una teoría para la enseñanza o la instrucción» (Kilpatrick, 1990, p. 42), es bastante frecuente que personas que se identifican con el constructivismo, incluso con versiones radicales, eludan la problemática filosófico-epistemológica y sostengan fundamentalmente un planteamiento pedagógico como núcleo de su constructivismo.

Es discutible que, a partir de los principios epistemológicos constructivistas, puedan plantearse consecuencias 
pedagógicas claras y exclusivas de este paradigma, por lo que es comprensible la resistencia apuntada a considerar una pedagogía constructivista. Pero en la práctica, y a veces con alusiones a una fundamentación epistemológica de tipo radical, en la literatura educativa de los últimos doce años predomina un enfoque «constructivista» de la enseñanza con algunos rasgos tajantes y dogmáticos, incluso oficializados gubernamentalmente. Así, el Ministerio de Educación y Ciencia español (1989, p. 31) enmarca «los principios psicopedagógicos que subyacen al Diseño Curricular Base» en «una concepción constructivista del aprendizaje escolar y de la intervención pedagógica, entendida en sentido amplio...», orientación constructivista que, en su desarrollo en la literatura logsiana, incluso en casos de adscripción al constructivismo social, mantiene conexiones con el radicalismo epistemológico constructivista.

Dentro de la corriente pedagógica constructivista, ocupa un lugar destacado la noción de ideas previas, diferenciada de la de conocimientos previos, como consecuencia de la impronta de los principios epistemológicos del constructivismo radical -diferencia no siempre percibida por algunos defensores del constructivismo.

La importancia de los conocimientos previos ha sido resaltada tradicionalmente por diversas teorías educativas (Miras, 1993, p. 48); de hecho, las secuencias establecidas en distintos programas educativos responden a un criterio sobre el carácter previo de unos conocimientos respecto a otros. Merece destacarse, como muestra de lo inadecuado de ciertos ataques simplistas contra el conductismo, la propuesta de Thorndike (1922, p. 199) sobre el valor de los conocimientos previos:

«Un plan científico para enseñar aritmética debería empezar con un inventario exacto del conocimiento y la habilidad que ya poseen los alumnos.»

Sin embargo, la importancia de las ideas previas, no en tanto que conocimientos, más o menos acertados, sino como expresión del individuo, como construcción personal, debe su actual reconocimiento al empeño en ese sentido del movimiento constructivista, especialmente de la línea radical. La desvinculación constructivista del conocimiento respecto a una realidad objetiva exterior propicia que las ideas de una persona adquieran valor al margen de su corrección o correspondencia con una realidad. Aunque no se compartan los principios del constructivismo radical, es innegable la repercusión positiva de esta tendencia epistemológica con ramificaciones educativas, en la medida en que se propone la valoración de opiniones no ajustadas a los moldes establecidos como inmutables y a la rigidez dogmática de algunas transposiciones didácticas.

El respeto y el realce de las ideas previas, sean éstas acertadas o no, no puede considerarse, sin embargo, como sucede con otros «temas» (Goldin, 1990, p. 32) un planteamiento exclusivamente vinculado a unos principios constructivistas radicales. El relativismo adjudicado por Berkeley (1710/1985, p. 129-130) al realismo, justifica el que incluso desde una posición materialista pueda defenderse la importancia de las ideas previas, dado el carácter siempre inacabado y revisable del conocimiento científico y que, en consecuencia, merezcan consideración las ideas no coincidentes con las admitidas -provisionalmente-como correctas.

Una situación similar de doble compatibilidad (con el constructivismo radical y con el realismo epistemológico) se produce también respecto a otros conceptos de interés psicopedagógico, como los relacionados con el procesamiento de la información (calificado por diversos autores como tendencia neoconductista) y con los planteamientos materialistas vygotskianos (Vygotsky, 1991, p. 200; Wertsch, 1988, p. 28), posteriormente incorporados al constructivismo social, como «pilar psicológico del mismo» (Weinberg y Gavelek, 1987, p. 346).

Reconocida la aportación para el aprendizaje que supone la valoración de las ideas previas, y no sólo de los conocimientos previos, es preciso señalar la existencia de sombras y dificultades en la concepción constructivista de las ideas previas, algunas de ellas, paradójicamente, dignas del más ingenuo y simple de los realismos.

La relatividad de la identificación de una idea como previa desmantela gran parte de la sacralización que de las ideas previas se ha realizado por parte de la doctrina constructivista, al poner en cuestión la pretendida naturaleza idiosincrásica de la generalidad de las mismas, como emanación del yo, al margen de contingencias exteriores.

El mismo Piaget ha intervenido en esta desmitificación de las ideas previas, al señalar «cinco tipos de reacciones revelados por examen clínico», distinguiendo los casos de «respuesta al azar» (llamada por Binet y Simon le n'importequisme)», de «fabulación», «convicción sugerida», «convicción liberada»y «convicción espontánea» (Piaget, 1973, p. 21-22).

La existencia de ideas arraigadas, resistentes incluso a su modificación mediante la enseñanza, distinguiéndolas de ideas superficiales (Martínez Delgado, 1998), y el proceso de formación de unas y otras, hasta el punto de que puedan considerarse tanto ideas previas como ideas resultantes, ofrece un importante campo de investigación educativa, en el que pueden ser contraproducentes algunos simplismos instruccionales.

El apriorismo de considerar el conjunto de las ideas previas como expresión sólo de tendencias internas del individuo, sin ninguna influencia moldeadora del medio exterior (cuya propia existencia y valor epistemológico se cuestiona por el constructivismo radical) supone englobar la historia de las diferentes ideas dentro de la indescifrable «caja negra del universo» (Glasersfeld, 1987, p. 108), en que el constructivismo coloca el mundo objetivo exterior a los individuos y las relaciones mutuas sujeto-objeto. Es sorprendente y paradójico, sin embargo, que, desde la perspectiva de los principios radicales, se pretenda averiguar las ideas previas de otras personas, cuando las personas mismas, y por lo tanto sus ideas, son 
creaciones propias de cada individuo, que no podría atribuir con pretensiones de objetividad (o de viabili$d a d$ ) sus propias creaciones a otras personas; por ejemplo, el profesor no podría pretender conocer las ideas previas de sus alumnos, como tales ideas propias de éstos, como recomienda el constructivismo pedagógico.

En conexión con los problemas de coherencia teórica entre los principios constructivistas generales y las fórmulas teóricas relativas a las ideas previas, se ignora con frecuencia, en las directrices pedagógicas de orientación constructivista, la dificultad de acceder a las mismas y de actuar sobre ellas, y se propaga la «presunción implícita» de que «descubrir qué piensa un niño es una tarea relativamente sencilla (Johnson y Gott, 1996, p. 562).

La rotundidad con que desde algunas zonas académicas y administrativas, de tendencia logsiana, se prescribe la averiguación de las ideas previas para una nueva enseñanza eliminadora del fracaso escolar, parece reflejar la misma seguridad en las posibilidades de llegar a un conocimiento pleno, objetivo y verdadero que la que se atribuye al conductismo, seguridad que parece particularmente problemática si tenemos en cuenta que se refiere a procesos internos del sujeto.

El hecho de que el constructivismo radical desplace la caja negra en que el conductismo (comportándose como un realismo parcial) encierra el mundo subjetivo del individuo, desde dicha subjetividad, al mundo de la realidad exterior, denota un cierto dualismo entre las dos teorías (conductista y contructivista), en la línea del hipotético isomorfismo realismo-constructivismo que desde el punto de vista de ciertas operaciones formales hemos bosquejado.

Si la noción de ideas previas, desde un enfoque constructivista radical, presenta en el terreno de la enseñanza-aprendizaje contradicciones conceptuales y prácticas, también encuentra problemas cuando se aplica a la investigación científica, donde revisten, en parte, la forma de marco teórico de investigación. Aunque entre el proceso de investigación y la labor de aprendizaje no existe un foso insalvable, al menos desde el punto de vista del aprendizaje por descubrimiento (en una connotación realista) o del aprendizaje por invención (sobre bases constructivistas), es interesante resaltar algunas formulas características de planteamientos constructivistas acerca de la investigación educativa. También en este campo se manifiesta con claridad la ilimitada y paradójica confianza, por parte de sectores constructivistas, en la trascendencia y objetividad del marco teórico declarado de una investigación. Y ello a pesar de que la declaración de principios acerca de la investigación emprendida se reduzca a veces a una fórmula ritual, de mayor eficacia si se realiza a favor de la corriente constructivista (o del paralelo reformismo LOGSE). La celeridad de algunas conversiones al constructivismo puede contribuir a explicar algunas divergencias entre el encuadre teórico declarado y los propios planteamientos teóricos aparentemente derivados, junto a la práctica concreta de la investigación.
La dirección de la revista Enseñanza de la Ciencias (3-10-1997), manifestó ante un trabajo presentado a su consideración:

«Pensamos, de acuerdo con muchos autores: que no existen trabajos empíricos que no estén sometidos a encuadramientos teóricos (toda observación está condicionada por la teoría), por lo que es importante precisar y argumentar qué referente psicológico o epistemológico ha orientado el trabajo.»

En esta formulación cabe distinguir dos aspectos: por un lado, la afirmación de la inevitable presencia de un substrato teórico en todo trabajo empírico, fórmula en principio trivial, aunque quizás sea discutible que el encuadramiento sea de carácter exclusivamente, o incluso predominantemente, teórico; y, por otro lado, la recomendación de que dicho marco previo deba ser precisado y argumentado.

La connotación teórica, incluso ideológica y de vinculación a intereses, de la actividad humana, incluida la investigación, es reconocida de forma general, pero el análisis del alcance de dicha connotación teórica merece toda clase de cautelas para no caer en un determinismo ramplón y en los excesos que históricamente se han producido en la calificación ideológica de distintas ideas u opiniones. De la misma forma que desde un punto de vista realista la hipótesis de la existencia objetiva de un mundo exterior influyente en nuestras concepciones no permite asegurar que determinada posición corresponde infaliblemente a unos u otros hechos objetivos, tampoco aquí resulta aceptable un dogmatismo interpretativo que permita adscribir de forma indiscutible cualquier investigación a unos principios determinados. Es más, la determinación del marco teórico ni siquiera es algo fácil ni seguro para el mismo autor de las opiniones y actividades; hay razones para pensar incluso que la autocalificación puede ser menos fiable que la realizada por otras personas.

A este respecto, y como una invitación a la reflexión sobre los principios básicos del constructivismo radical, son reveladores los versos de Louis Aragon:

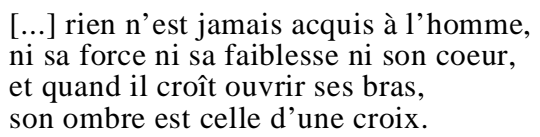

Una vez más nos encontramos con la paradoja de que el constructivismo, partiendo de un principio general que invita al relativismo, se excede en sus pretensiones de llegar a un conocimiento seguro y verdadero, precisamente en los terrenos en que, desde una perspectiva realista es aconsejable la mayor prudencia.

Las críticas dirigidas anteriormente al enfoque constructivista radical de las ideas previas relacionadas con la naturaleza, se agigantan cuando nos referimos a fenómenos psicológicos o sociales, cuya complejidad dificulta aún más los dictámenes terminantes y definitivos. 
Por otra parte, admitiendo la posibilidad de que el marco teórico, de una actividad o de una investigación, pueda precisarse razonablemente, la exigencia de que dicho encuadre sea formulado explícitamente parece excesivo porque, a pesar de algunas apariencias expositivas, el fondo de una investigación no consiste meramente en un proceso deductivo a partir de los axiomas teóricos y porque debería respetarse el derecho de los autores a llevar su declaración teórica hasta donde éstos crean conveniente plantearla, incluso a despreciar planteamientos considerados como excesivamente teóricos, en consonancia con la manifestación de Montessori: «No me gustan los fílósofos. A mi sólo me inspira la realidad» (citado por Moreno, Poblador y Río, 1984, p. 455).

El requerimiento para que, puesto que existen, se formulen los marcos teóricos de una investigación se inserta, en general, en el contexto de una relación asimétrica entre el organismo de dirección (y sus asesores) y el investigador autónomo, relación que tiene algunas características y cierta proximidad con las relaciones de poder. Esta asimetría se refleja, entre otras facetas, en que, mientras los planteamientos de una investigación se consideran inevitablemente unidos a un marco teórico, incluso a una finalidad, las decisiones de la dirección de una revista, o de otra institución selectiva, gozan de una presunción especial de objetividad y de calidad.

Sin pretender detectar de forma terminante las líneas seguidas por uno u otro grupo directivo ni insertar inequívocamente los aspectos teóricos entre las consecuencias de determinadas formas de estructuración social, proponemos la reflexión sobre algunas conjeturas explicativas generales.

Si bien, dentro de los criterios empleados por grupos encargados de canalizar y promover la investigación científica (publicaciones científicas, tribunales de selección...), puede reivindicarse la defensa de criterios evaluadores de cierta objetividad (relativa), cuya desaparición produciría probablemente algunos trastornos, cabe plantear también la coexistencia de estos elementos positivos con otros componentes, estructurales y no sólo, ni fundamentalmente, personales, que obedecerían a la defensa de intereses corporativos y que desviarían la inicial objetividad reclamada hacia la paralización de la investigación libre.

Entre las funciones de tipo corporativista puede contemplarse la actividad eliminadora y distanciadora de trabajos procedentes del exterior de la casa. Para ello puede servir de señal la mayor o menor disposición y pomposidad para alinearse con posiciones teóricas imperantes y para enfeudarse respecto a grupos más o menos estructurados que controlan al menos parte de la producción y distribución de, en este caso, la investigación educativa (dentro de lo que podríamos llamar nomenklatura académica).

Con la crítica anterior, sin embargo, no se pretende elevar a virtud la carencia sistemática de referentes teóricos o el desconocimiento de trabajos próximos al realizado, aunque la profundidad conceptual quizás deba tener más peso que ciertas liturgias más propias de comportamientos sacerdotales que del ideal científico.

Aunque el concepto de ideología haya perdido vigor recientemente, quizás no sea del todo descartable la vinculación entre marco teórico, de una investigación o de una decisión valorativa, e ideología, ideología que en sus grandes direcciones reflejaría la defensa de bloques de intereses. A la vinculación entre postura teórica e ideología, y entre ésta e intereses, no es atribuible una naturaleza rígida y estable, y menos aún una transparencia que permita el enjuiciamiento tajante y definitivo; eso no impide, sin embargo, la discusión de conjeturas e hipótesis que arrojen alguna luz sobre estas complejas cuestiones.

Nuestra crítica se dirige, pues, tanto contra los excesos de fe en la vinculación entre marco teórico e investigación práctica, como contra el estancamiento en el análisis de la conexión de una investigación con factores externos a los «datos» de la misma, sin que vaya más allá de la pantalla de un presumible marco teórico y sin que se susciten posibles condicionamientos ideológicos y grupales.

\section{EL PROBLEMA DE LOS MÉTODOS EMPÍ- RICOS Y EXPERIMENTALES DE APRENDI- ZAJE Y DE INVESTIGACION CIENTÍFICA}

Ya hemos expuesto cómo la noción de ideas previas presenta problemas en cuanto al propio concepto y en cuanto a la justicia de su adscripción exclusiva al modelo constructivista de conocimiento. Ahora dedicaremos alguna atención a analizar la actitud del constructivismo radical respecto a los métodos empíricos y experimentales de aprendizaje e investigación.

En principio no existe una contradicción de base entre los postulados del constructivismo radical y los métodos empíricos y experimentales utilizados en la enseñanza y en la investigación científica (incluido el método inductivo). Por el contrario, sería de esperar que, dada la importancia concedida por el constructivismo al mundo experiencial del sujeto, las vivencias empíricas, y más aún las dirigidas intencionalmente por el propio individuo, de modo activo, en forma de experimentación, recibieran el apoyo de la concepción constructivista del conocimiento; en la misma dirección apuntaría el propósito constructivista de conservar los resultados científicos, difícilmente rechazables por cualquier teoría que no quiera verse reducida a la marginalidad de una secta. $\mathrm{Si}$ acaso, se podría esperar que el constructivismo reinterpretara, de acuerdo con su propia epistemología, estos procedimientos, empíricos y experimentales, de investigación y de aprendizaje, advirtiendo del peligro objetivista. De hecho, sin embargo, se viene produciendo un rechazo, por parte del constructivismo, de los métodos empíricos de aprendizaje e investigación, subrayando así que «la teoría constructivista del conocimiento y la práctica constructivista de la enseñanza están demasiado 
distantes y dejan demasiado espacio vacío en medio» (Schoenfeld, 1992, p. 294).

Con una cierta caricaturización de los planteamientos contrarios, Porlán (1988, p. 201) sostiene, en una perspectiva constructivista de la enseñanza y de la ciencia:

«En el mejor de los casos se plantea la búsqueda "en la realidad" de las explicaciones conceptuales: las observaciones en el medio y las experiencias de laboratorio llevarán, por sí mismas al alumno al aprendizaje "correcto" de los conceptos científicos acabados. En ningún caso se expresan referencias al pensamiento propio del niño, sus concepciones previas, sus representaciones sobre la realidad [...] En general, los sujetos de la muestra apuestan por un modelo de producción científica de conocimientos del tipo observación-hipótesisexperimentación-teoría, por una concepción del aprendizaje científico basado en el niño con "mente en blanco" o "vaso vacío", y por unos métodos de enseñanza coherentes con lo anterior (hay que partir de la observación de la realidad para llegar al conocimiento).»

De igual forma se pronuncian Pope y Scott (1983, 1988, p. 181) al anunciar su observación, en su trabajo con educadores, de que «la concepción positivista, empiroinductivista de la ciencia está en consonancia con una visión absolutista de la verdad y del conocimiento [...]».

La repulsa hacia los métodos empíricos de investigación se expresa, de forma contradictoria, en la objeción comunicada por la dirección de la revista Enseñanza de la Ciencias (3-10-1997) a una investigación:

«[...] el problema del artículo no son las críticas al conductismo o al constructivismo vertidas en él, sino la defensa implícita que se hace del empirismo en la investigación psicológica y didáctica».

Aparte de las reflexiones ya realizadas sobre la cuestión de hacer explícito (o dejar implícito) el marco teórico que afecta de forma más o menos pronunciada una investigación, se suscita la cuestión de qué se entiende por defensa implícita del empirismo. Si, como sería acorde con otros textos constructivistas, se desaprueba la utilización de métodos empíricos de investigación que no vaya acompañada de una declaración de adscripción a la filosofía empirista, nos encontraríamos ante una rigidez teórica rayana al dogmatismo. Piaget, reivindicado por constructivistas radicales como Glasersfeld, como el padre de este paradigma, sostuvo:

«[...] gran número de ejemplos muestran que se puede ser psicólogo estrictamente experimentalista e interpretar la formación de los conocimientos de forma antiempirista o independiente de la filosofía empirista [...]» (Piaget, 1965, 1992, p. 78).

La oposición al empleo de métodos empíricos y experimentales de investigación y la plena inserción de los mismos en el marco de la filosofía empirista exigirían la presentación de alternativas a dichos procedimientos que no se reduzcan a disquisiciones nominales y escolás- ticas sobre el empirismo; estas alternativas, con la salvedad parcial de la inclinación por los métodos cualitativos y por los deductivos, no cristalizan de forma suficientemente clara.

La asignación de procedimientos de indagación (empíricos, experimentales, de introspección) a unos u otros principios filosóficos parece anunciar una predisposición a favor de los métodos deductivos de investigación, con carácter excluyente respecto a otras posibilidades. Lo inapropiado de considerar que el empleo de un método empírico conlleva, cuando menos implícitamente, la adopción de unos principios filosóficos empiristas es comparable a que, de forma automática, la utilización del método deductivo -cuyo valor y potencia son reconocidos especialmente en el pensamiento lógico y matemático- implicara la presunción de una concepción filosófica idealista y espiritualista.

Independientemente de sutilezas teoricistas, merece resaltarse que la apelación a los datos empíricos, a los hechos, a la observación sistemática y a la experimentación supuso un salto extraordinario frente al oscurantismo y la especulación escolástica anteriormente reinantes y que, quizás bajo otras formas, muestre cierta revitalización en la actualidad.

La peculiar relación del constructivismo radical con el empirismo y el positivismo, interpretados ambos como formas de realismo epistemológico (cuestión que resulta, como mínimo, discutible, al menos respecto a sus orígenes), puede seguirse a través de la relación entre la dirección académica de un programa (SCIIS) para la enseñanza de las ciencias en quinto grado de orientación constructivista y una profesora ejecutora del programa (Ms. Howe), con cuya inocencia y buena y sufrida voluntad no se puede sino expresar la mayor simpatía. La actividad de aprendizaje analizada por Smith y Anderson, (1988) se centra en la fotosíntesis, cuyo concepto «hay que "inventar", según la terminología de SCIIS [...] y usarlo para explicar los resultados» (p. 160).

La profesora, Ms. Howe, tomando en serio y rigurosamente la consigna constructivista, había intentado que los alumnos llegaran al concepto de fotosíntesis, sin conseguirlo. El fracaso del intento se resuelve por parte del equipo directivo imputando un desviacionismo epistemológico a la profesora y, al mismo tiempo, debilitando la radicalidad del propio paradigma constructivista. Como la profesora intentó llevar a los alumnos hacia la construcción del concepto de fotosíntesis, por «la observación empírica de plantas que crecen a la luz y en la oscuridad», sentencian los autores:

«[...] las creencias de Ms. Howe eran coherentes con la posición filosófica del positivismo lógico, que defiende que las teorías científicas son inferidas a partir de datos por medio de una lógica inductiva» (p. 168).

Y como esta actitud inductivista de la profesora no parece suficiente para explicar el fracaso, los directivos rebajan los propios principios y responsabilizan nueva- 
mente, de la rigidez, a la sufrida Ms. Howe, mediante el siguiente retruécano:

«[...] el no presentar a los estudiantes una definición y explicación de la fotosíntesis, tal como se sugería, constituye una segunda desviación [...] En el punto en que Ms. Howe planteó el interrogante, la guía del profesor SCIIS requiere que se presente a los estudiantes el concepto de fotosíntesis. El sugerir esto refleja el «ciclo de aprendizaje» SCIIS en el que los nuevos conceptos explicativos son «inventados» (claramente es el profesor quien los presenta) después de la «exploración» que los estudiantes hacen, en la que desarrollan observaciones y preguntas adecuadas [...] El punto de vista SCIIS, que se corresponde con el de los filósofos Khun y Toulmin, es que estos conceptos son invenciones creativas que no es probable que se les ocurran a los estudiantes» (p. 169-170).

La labilidad y carácter acomodaticio de los planteamientos ( sólo firmes en la condena de la actitud de Ms. Howe, salvo amplificación debida a la traducción), llegando a admitir la denostada enseñanza transmisiva, presenta tonos sarcásticos y pone en duda la coherencia de los principios constructivistas. Lo que sí queda claro es la asimetría, característica de las relaciones de poder y dominación, que impregna la relación entre el equipo dirigente de la experimentación y la profesora ejecutante, en la que los fracasos sistemáticamente son producto de los errores propios de los subordinados o de falsas interpretaciones de éstos, y nunca de errores teóricos atribuibles a los grupos dirigentes: Un dogmatismo tradicional en la justificación de distintas modalidades de autoritarismo, un tanto reñido con la hipótesis inicial del constructivismo radical de que no existe una verdad objetiva más allá del individuo.

Algunas situaciones angustiosas de profesores de matemáticas y de ciencias - motivadas por la credulidad en la publicidad de la LOGSE en el sentido de hacer desaparecer el fracaso escolar, que el aprendizaje de los alumnos sea espontáneo y sin esfuerzo, y de eliminar las desigualdades y discriminaciones sociales preexistentes entre los alumnos- pueden proceder de la interiorización de una presunción jerarquizadora que asigna, de forma automática e inflexible, el acierto al dirigente y el error al subordinado, como se refleja en la narración referida del programa SCIIS.

El rechazo por el constructivismo de los métodos empíricos y experimentales que, al igual que sucede respecto a los planteamientos realistas, una vez arrojados reaparecen sistemáticamente dentro de la misma doctrina que los expulsa suscita no sólo un problema referente al método de aprendizaje o de investigación, sino también el problema teórico de las proximidades y diferencias entre la filosofía empirista y los principios del constructivismo radical.

Debe destacarse una plena coincidencia inicial entre empirismo y constructivismo, respecto a negar la existencia de un mundo objetivo, exterior al sujeto, o cuando menos que ese posible mundo exterior pueda moldear nuestros conocimientos. Así, Hume (1984, p. 328), en frase que nos recuerda el principio distintivo del constructivismo radical respecto al constructivismo trivial y la distinción entre asuntos prácticos y asuntos científicos, asevera:

«En efecto, la filosofía nos dice que toda cosa manifiesta a la mente no es sino una percepción y que ésta es discontinua y dependiente de la mente; el vulgo, en cambio, confunde percepciones y objetos, y atribuye una existencia distinta y continua a las cosas que ve y siente.»

En cierto sentido puede afirmarse que la posición teórica del empirismo de Hume es, al menos en sus formulaciones iniciales, más radical y más coherente que la adoptada posteriormente por el constructivismo radical. Russell (1979, p. 634) manifiesta que «Hume [...] representa, en cierto sentido, un punto final: es imposible ir más allá en su dirección». Las diferencias entre ambas posiciones filosóficas proceden de esa diferente radicalidad en la negación de la existencia de sustancias metafísicas, que el empirismo lleva hasta la negación del yo (en el libro I del Tratado de la naturaleza humana, de Hume, aunque en los dos libros siguientes, se reintroduzca dicha idea del yo), quedando como base del conocimiento la colección de percepciones, mientras que el constructivismo defiende, sin titubeos ni dilaciones, la existencia de un yo, creador de las percepciones y de la experiencia. De esa forma, el constructivismo aparece con una más decidida vocación metafísica que el empirismo de Hume, evidenciada en la idea de un yo dotado de racionalidad.

La diferencia expuesta, en el distinto grado de metafisicación del sujeto, se invierte por parte del constructivismo radical, repudiando como metafísica la metodología empírica (metodología no exclusiva del empirismo filosófico y asumible incluso, en nuestra opinión, por el pensamiento constructivista radical).

Hemos abordado hasta ahora dos aspectos básicos de la enseñanza constructivista, las ideas previas y el rechazo de los métodos empíricos y experimentales de enseñanza, aprendizaje e investigación (inductistas y base de la enseñanza por descubrimiento), a los que se sustituye por el aprendizaje por invención, invención respecto a la que es pertinente recordar como muestra de los obstáculos para conseguir una coherencia constructivista, la opinión (cuyo excesivo empirismo y elitismo puede ser discutible) de Mach (1976, p. 56), -considerado un precursor del constructivismo radical- sobre las limitaciones de la invención como método de aprendizaje:

«En cualquier caso, muy pocas personas son inventores, la mayoría de los otros usa y aprende lo que esos pocos han inventado: ésa es la esencia de la educación...»

Otros dos enfoques y técnicas de enseñanza de las ciencias, de valor reconocido, han sido absorbidas por la corriente constructivista, la resolución de problemas (con insistencia en el tipo abierto de problemas), estrechamente vinculada a las ideas previas y a la enseñanza 
por invención (enseñanza centrada en el sujeto), y el aprendizaje por esquemas, como los mapas conceptuales, instrumentos para «representar un conjunto de significados conceptuales, incrustados en un armazón de proposiciones» (Novak y Gowin, 1984, p. 15). En la utilización de ambos métodos por el constructivismo radical pueden encontrarse tanto aportaciones positivas como censurables, aunque en este trabajo no entraremos en su análisis.

\section{ALGUNAS CONCLUSIONES Y PERSPEC- TIVAS}

Como resumen del análisis realizado y de algunas consecuencias deducibles del mismo proponemos reflexionar sobre las siguientes conclusiones y perspectivas, algunas de las cuales afectan al panorama general epistemológico, otras a la enseñanza y a la investigación científica y alguna a las relaciones entre teóricos de la educación y dirigentes administrativos, por un lado, y, por otro, profesores ejecutantes y con la responsabilidad de hacer progresar el conocimiento de los alumnos en el trabajo diario.

1) Defensa de los métodos empíricos y de la experimentación en la enseñanza-aprendizaje y en la investigación. Como señaló Piaget (1975/1983, p. 51):

«La crítica del empirismo no consiste en negar el papel de la experimentación; no obstante, el estudio "empírico" de la génesis del conocimiento muestra de entrada la insuficiencia de la interpretación "empirista" de la experiencia. No hay conocimiento alguno que deba su origen únicamente a las percepciones, porque éstas están siempre dirigidas y encuadradas por esquemas de acciones.»

Esta defensa de los métodos empíricos y experimentales debe evitar caer en la plena identificación entre los métodos de aprendizaje (donde los conocimientos asentados permiten atajos, evitando la repetición de los esfuerzos de los primeros descubrimientos) y los métodos de investigación de nuevos conocimientos (Ogborn, 1994, citado por Osborne, 1996, p. 67). El aprendizaje por investigación no debe, por lo tanto, pretender el monopolio de las actividades de enseñanza y aprendizaje.

2) Utilización de la enseñanza transmisiva, tampoco en este caso con propósitos excluyentes, realizable de forma significativa y no necesariamente memorística (Ausubel, Novak y Hanesian, 1983), aunque el cultivo de la memoria deba merecer también consideración.

3) Prestar atención y respeto a las ideas previas de los alumnos, apoyarse en ellas o inducir conflictos cognitivos (quizás más justificados en una perspectiva realista que en la constructivista radical) pero sin presunciones superficiales y aventuradas ( «el supuesto de que el niño "tiene ideas" no es suficiente base para conceder tal estatus a cualquier respuesta» (Johnson y Gott, 1996, p. 563).
4) Valoración de técnicas de trabajo como los esquemas, mapas conceptuales y en general las aportaciones sobre relaciones productivas y fundamentales en la conexión entre conceptos, pero evitando caer en una nueva forma de memorismo esquematista y en rituales permanentes de formación de mapas conceptuales, que pueden tener la misma superficialidad y embrollo que cualquier otra forma de exposición y de síntesis.

5) Crítica de algunas formas de «investigación» que, so pretexto de carácter cualitativo y de no caer en algunos excesos dogmáticos de la experimentación, desvirtúan el método científico y caen en el nuevo dogmatismo de presentar como resultados definitivos lo que son constructos banales e impresiones puramente subjetivas, contaminadas en ocasiones por la técnica triunfalista de la propaganda.

6) La exigencia de un determinado nivel de claridad en la investigación y en su comunicación, la conveniencia de reflexión sobre el marco teórico, las ideologías previas, las connotaciones y consecuencias de tipo teórico y práctico no deben conducir a la imposición de una declaración explícita sobre el marco teórico de trabajos de investigación, declaración que, por otra parte, pueda guardar escasa cercanía con la propia investigación.

7) Aumento de la consideración hacia los realizadores prácticos en la investigación educativa, planteando una colaboración en la que las mutuas aportaciones no se limiten a la labor de diseño por parte del investigador oficial y a la ejecución por parte del profesor. A pesar de la publicidad de la idea de investigación en el aula, la realidad de la investigación educativa presenta indicios de excesiva subordinación de los prácticos al teórico investido como tal. Paralelamente es reivindicable un mayor respeto hacia la labor cotidiana de los profesores, evitando las vistosas y provocativas consignas del tipo de «la mejor forma de enseñar es no enseñar» (Lange, director del Instituto Freudenthal, citado por Reeuwijk, 1992, p. 516) y algunos excesos publicitarios constructivistas.

8) Revalorización del realismo como epistemología. Russell (1976, p. 107), al referirse al solipcismo («doctrina de que no existe razón válida ni para afirmar ni para negar, excepto las propias experiencias»), sostiene: «[...] no creo que esta teoría pueda ser refutada, pero tampoco creo que nadie pueda creer en ella sinceramente». La indemostrabilidad de planteamientos paradigmáticos, la necesaria adaptación de éstos a éxitos reconocidos como los de la ciencia, incluso la idea de isomorfismo que hemos bosquejado, conducen a no plantear el enfrentamiento entre paradigmas en términos de exterminio teórico de uno u otro, aunque cada cual encuentre razones y argumentos en favor de uno y en contra del otro paradigma. En nuestro caso, hemos argüido en contra de la hegemonía del constructivismo y a favor del realismo epistemológico, aceptado de forma general tanto en la vida práctica como en la actividad científica, como el mismo Glasersfeld reconoce. La cíclica recaída de planteamientos inicialmente no realis- 
tas en el objetivismo exterior es una muestra de la renuencia de éste a desaparecer y de la potencia del modelo. En cualquier caso, es de desear que la relatividad que el constructivismo predica del conocimiento en

\section{REFERENCIAS BIBLIOGRÁFICAS}

ALTHUSSER, L. (1975). Curso de filosofía para científicos. Barcelona: Laia.

AUSUBEL, D.P., NOVAK, D.J. y HANESIAN, H. (1983). Psicología educativa. Un punto de vista cognoscitivo. (2a. ed.). México: Interamericana.

BERKELEY, G. (1710/1985). Principios del conocimiento humano. Madrid: Sarpe.

ERNEST, P. (1989). The constructivist perspective, en Ernest, P. (ed.). Mathematics Teaching. The State of the Art. Nueva York: The Falmer Press.

ERNEST,P.(1996). Varieties of constructivism: Their metaphors, epistemologies and pedagogical implications, en Fox, R. (ed.). Perspectives on constructivism (56). Media and Ressources Centre. University of Exeter, School of Education.

ETCHENBERGER, M.L.y SHAW, K.L.(1992). Teacher Change as a Progression of Transitional Images: A Chronology of a Developing Constructivist Teacher, School, Science and Mathematics, 92(8), pp. 411-417.

GLASERSFELD, E. VON (1987). Wissen, Sprache und Wirklichkeit. Arbeiten zum radikalen Konstructivismus. Braunschweig/Wiesbaden: Friedr. Vieweg \& Sohn.

GLASERSFELD, E. VON (1990). An Exposition of Constructivism: Why Some Like It Radical, en Davis, R. B., Maher, C. A. y Noddings, N. (eds.). Constructivist Views on the Teaching and Learning of Mathematics, pp. 19-29. National Council of Teachers of Mathematics (NCTM). Reston, Virginia.

GLASERSFELD, E. VON (1991). Constructivism in Education, en Lewy, A. The International Encyclopedia of Curriculum. Oxford: Pergamon Press.

GLASERSFELD, E. VON (1993, ed. alemana de 1981). Introducción al constructivismo radical, en Watzlawick, P. et al. La realidad inventada. Barcelona: Gedisa.

GLASERSFELD, E. VON (1995). Radical Constructivism: A way of knowing and learning. Londres: The Falmer Press.

GLASERSFELD, E. VON (1996). Footnotes to «The Many Faces of Constructivism». Educational Researcher, 25(6), p. 19.

GOLDIN, G.A. (1990). Epistemology, Constructivism and Discovery Learning in Mathematics, en Davis, R.B., Maher, C.A. y Noddings, N. (eds.). Constructivist Views on the general se aplique también a la valoración de otros modelos teóricos y prácticos de enseñanza-aprendizaje y de investigación, discrepantes con los oficializados como constructivistas.
Teaching and Learning of Mathematics. National Council of Teachers of Mathematics. Reston, Virginia, pp. 31-47.

GÓMEZ-GRANELL y COLL SALVADOR C. (1994). ¿De qué hablamos cuando hablamos de constructivismo? Cuadernos de Pedagogía, 221, pp. 8-10.

HUME, D. (1734/1984). Tratado de la naturaleza humana. Barcelona: Orbis.

JOHNSON, PH. y GOTT, R. (1996). Constructivismand Evidence from Children's Ideas. Science Education, 80(5), pp. 561-577.

KILPATRICK, J. (1990). Lo que el constructivismo puede ser para la educación de la matemática. Educar, 17, pp. 37-52.

MACH, E. (1976). Knowledge and Error. Sketches on the Psychology of Enquiry. Dordrecht: D. Reidel Publishing.

MARTÍNEZ DELGADO, A. (1995). Constructivismo, ¿una vuelta a los principios filosóficos del positivismo? Comunicación, Lenguaje y Educación, 28, pp. 5-13.

MARTÍNEZ DELGADO, A. (1998). Ideas previas: experimentación acerca de ideas arraigadas e ideas inducidas sobre fracciones. Suma, 28, pp. 59-70.

MARX, K. (1845/1975). Obras escogidas (2 tomos). Madrid: Akal.

MIRAS, M. (1993). Un punto de partida para el aprendizaje de nuevos contenidos: los conocimientos previos, en Coll, C., Martín, E., Mauri, T. et al. El constructivismo en el aula. Barcelona: Graó.

MORENO G.J.M., POBLADOR, A. y RÍO, D. DEL (1984). Historia de la educación. Madrid: Paraninfo.

NODDINGS, N. (1990). Constructivism in Mathematics Education, en Davis, R.B., Maher, C.A. y Noddings, N. (eds.). Constructivist Views on the Teaching and Learning of Mathematics, p. 7-18. National Council of Teachers of Mathematics (NCTM). Reston, Virginia.

OGBORN, J. (1994). Theoretical and empirical investigations of the nature of scientific and commonsense knowledge. Tesis doctoral no publicada. Londres: King's College.

OSBORNE, J.F. (1996). Beyond Constructivism. Science Education, 80(1), pp. 53-82.

PIAGET, J. (1965/1992). Sagesse et illusions de la philosophie. París: Presses Universitaires de France. 
PIAGET, J. (1973). The Child's Conception of the World. Paladin, St. Albans.

PIAGET, J. (1975/1983). La psicogénesis del conocimiento y su significado epistemológico, en Chomsky, N. y Piaget, J. Teorías del lenguaje. Teorías del aprendizaje. Barcelona: Crítica.

POPE, M.L. y SCOTT, E.M. (1983/1988). La epistemología y la práctica de los profesores, en Porlán, R., García, J.E. y Cañal, P. (comp.). Constructivismo y enseñanza de las ciencias, pp. 179-191. Sevilla: Díada.

PORLÁN, R. (1988). El pensamiento científico y pedagógico de maestros en formación, en Porlán, R., García, J.E. y Cañal, P. (comp.). Constructivismo y enseñanza de las ciencias, pp. 193-203. Sevilla: Díada.

REEUWIJK, M. VAN(1992). The Standards Applied: Teaching Data Visualization. Mathematics Teacher, 85(7), pp. 513-518.

RUSSELL, B. (1976). La evolución de mi pensamiento filosófico. Madrid: Alianza.

RUSSELL, B. (1979). History of Western Philosophy. Londres: Unwin Paperbacks.

SAUNDERS, W.L. (1993). The Constructivist Perspective: Implications and Teaching Strategies for Science. School, Science and Mathematics, 92(3), pp. 136-141.
SCHOENFELD, A.H. (1992). Radical Constructivism and the Pragmatics of Instruction. Review of Radical Constructivism in Mathematics Education (E. von Glasersfeld). Journal for Research in Mathematics Education, 23(3), pp. 290-295.

SMITH, E.L. y ANDERSON, C.W. (1988). Las plantas como productores: Un estudio de caso en la enseñanza elemental de las ciencias, en Porlán, R., García, J.E. y Cañal, P. (comp.). Constructivismo y enseñanza de las ciencias, pp. 157-177. Sevilla: Díada.

THORNDIKE, E.L. (1922). The Psychology of Arithmetic. Nueva York: The Macmillan Company.

VYGOTSKY,L.S. (1991). Prólogo a la edición rusa del libro de W. Kohler «Investigaciones sobre la inteligencia de los monos antropomorfos», en Obras escogidas (tomo I). Madrid: Visor-MEC.

WEINBERG, D. y GAVELEK, J. (1987). A social constructivist theory of instruction and the development of mathematical cognition, en Bergeron, J.C., Herscovics, N. y Kieran, C. (eds.). Psychology of Mathematics Education. Proceedings of the International Conference, vol. III. Montreal.

WERTSCH, J.V. (1988). Vygotsky y la formación social de la mente. Barcelona: Paidós.

[Artículo recibido en diciembre de 1997 y aceptado en noviembre de 1998.] 